\title{
The endoclamp device as a useful strategy during redo surgery on the aortic root and arch
}

\author{
Giovanni Ruvolo ${ }^{1}$, Paolo Nardi ${ }^{1}$, Carmela Rita Balistreri ${ }^{2}$, Fabio Bertoldo ${ }^{1}$, Fernando Dionisio Colella ${ }^{1}$, \\ Calogera Pisano ${ }^{1}$ \\ ${ }^{1}$ Cardiac Surgery Unit, Tor Vergata University Hospital, Rome, Italy \\ ${ }^{2}$ Department of Pathobiology and Medical and Forensic Biotechnologies, University of Palermo, Italy
}

Kardiochir Torakochir Pol 2019; 16 (4): 209-211

The endoclamp device has been widely used in miniinvasive mitral valve surgery with very good results compared to the transthoracic clamp [1]. Some authors have underlined the safety and effectiveness of this device in complex and emergency operations [2]. We reported our experience during a redo operation related to a pseudoaneurysm that developed for total proximal anastomosis detachment after a classic Bentall operation.

A 19-year-old man with Marfan syndrome underwent a Bentall procedure due to a $55 \mathrm{~mm}$ aortic root aneurysm and severe bicuspid aortic valve regurgitation. Nine months later, he complained of fever, malaise and enteritis. Inflammatory markers were rising. Blood culture showed positivity of Staphylococcus hominis. Combination antibiotic therapy with gentamicin, ceftriaxone and vancomycin was started. An emergency transesophageal echocardiogram (TEE) and color Doppler mapping revealed the detachment of the valve conduit from the annulus and a large pseudoaneurysm. The prosthetic valve appeared to have normal motion and to be free of any vegetation. No aortic regurgitation was noted either (Figure $1 \mathrm{~A}$ ). Color-flow imaging showed the entrance of most of the cardiac stroke volume into a large pseudoaneurysm covering almost the entire circumference and length of the Dacron graft as far as it could be seen (Figure $1 \mathrm{~B}$ ). The computed tomography (CT) scan confirmed the TEE evidence (Figure $1 \mathrm{C}$ ). An urgent operation was planned. We decided to use an endoclamp device (EndoClamp Intra-aortic Occlusion Device, Edwards Lifesciences Corp); for this reason we inserted in the femoral artery an EndoReturn Arterial Cannula 21 Fr. The cardiopulmonary bypass was initiated prior to resternotomy with cannulation of the femoral vessels. After the resternotomy, massive bleeding from the right atrium happened. Immediately, the endoclamp device was inflated (Figure $2 \mathrm{~A}$ ), the heart was arrested using Custodiol cardioplegia. A huge pseudoaneurysm was detected and the valve conduit was found to be hanging above the aortic ring, fully detached and suspended in place by the coronary arteries (Figure $2 \mathrm{~B}$ ). Because the aortic annulus was completely destroyed by a previous endocarditis, we decided to implant a new valve conduit inside the left ventricular outflow tract (Figure $2 \mathrm{C}$ ). The postoperative period was uneventful and the patient was discharged home in good general condition.

This is new strategy to treat a giant pseudoaneurysm that developed for total proximal anastomosis detachment after a classic Bentall operation, which was successfully solved by the use of an endoclamp device. A prerequisite for this technique is the presence of an adequate site for the Endoclamp device inflation, preferably a Dacron graft, as in the present case. Moreover, the origin of the pseudoaneurysm must be well documented and be localized proximally to the site at which the Endoclamp device will be inflated. This technique obviates the use of profound hypothermia, thus reducing cardiopulmonary bypass (CBP) duration and its complications [3]. Currently, in the presence of a giant pseudoaneurysm closely related to the sternum, peripheral initiation of CPB with sternal opening during profound hypothermia is the technique most often advocated [4]. However, this technique is related to a long CPB duration. The duration of CPB seems to be an independent predictor of perioperative death for patients undergoing reoperation of the aortic root or ascending aorta [5] because it is related to numerous and dangerous complications. Consequently, redo operations on the ascending aorta and aortic arch are characterized by high morbidity and mortality. In order to reduce morbidity and mortality related to this operations, it is necessary to avoid profound hypothermia, reduce CPB duration and prevent complications related to technical complexity. In our opinion the Endoclamp device could be a useful and effective solution.

In conclusion, we advocate the use of the Endoclamp device not only for small thoracotomy mitral valve surgery but especially in the situation in which treatment is dif-

Address for correspondence: Calogera Pisano, Cardiac Surgery Unit, Tor Vergata University Hospital, Rome, Italy, phone: +39 3283297692 ,

e-mail: lindapisano82@gmail.com

Received: 20.08.2019, accepted: 13.10.2019. 

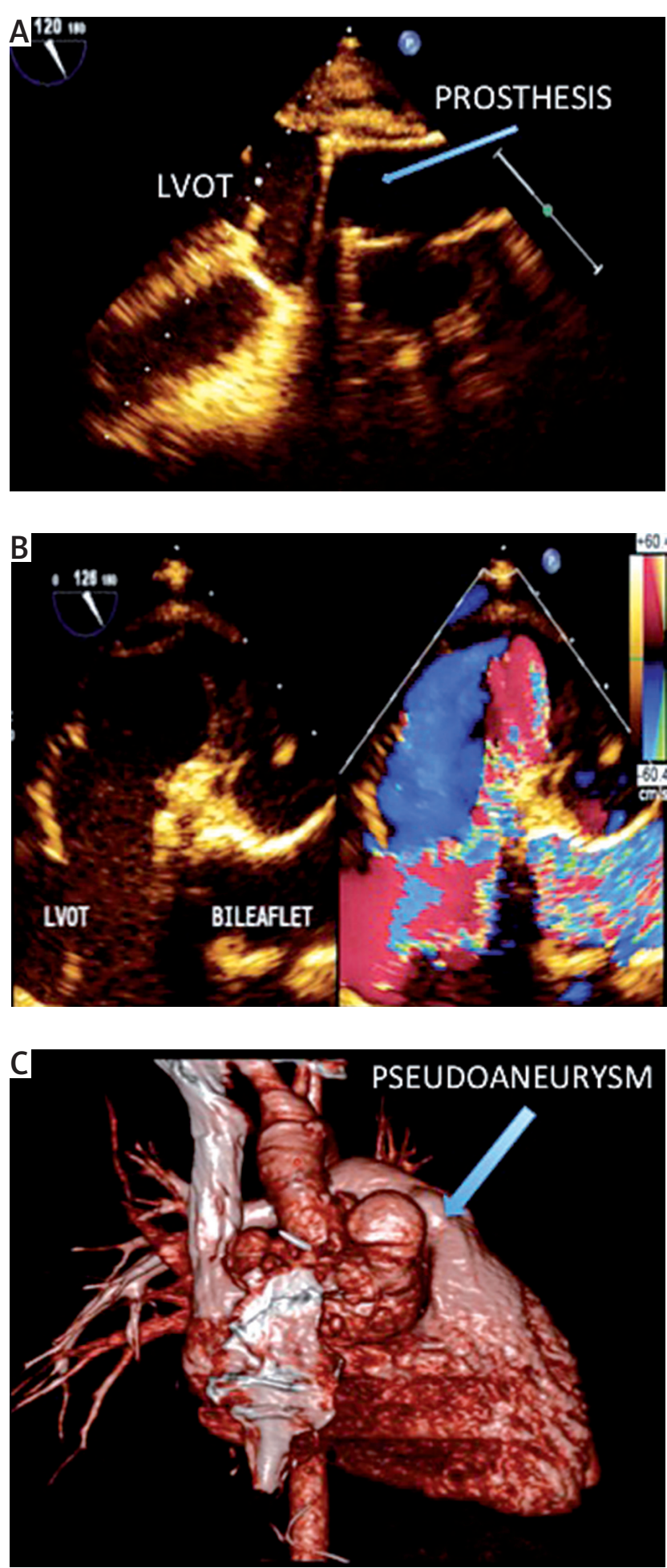

Figure 1. A - Transesophageal echocardiogram images. B - Echo color-flow images. C - CT scan images

ficult and requires a well-planned operative strategy such as a redo operation in the ascending aorta and aortic arch.

\section{Disclosure}

The authors report no conflict of interest.
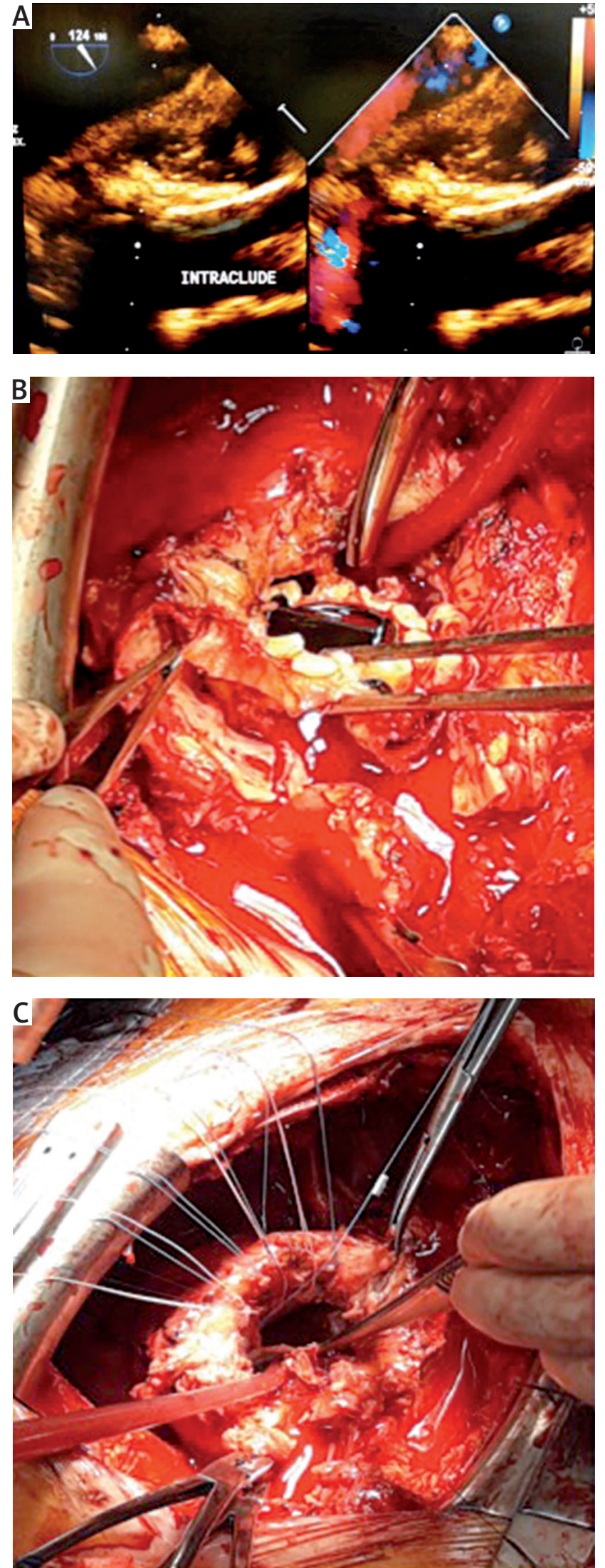

Figure 2. A - Transesophageal echocardiogram view of the endoclamp device. B - Intraoperative view of the fully detached valve conduit. C - New valve conduit implanted inside the left ventricular outflow tract 


\section{References}

1. Kowalewski M, Malvindi PG, Suwalski P, Raffa GM, Pawliszak W, Perlinski D, Kowalkowska ME, Kowalewski J, Carrel T, Anisimowicz L. Clinical safety and effectiveness of endoaortic as compared to transthoracic clamp for small thoracotomy mitral valve surgery: meta-analysis of observational studies. Ann Thorac Surg 2017; 103: 676-686.

2. Dagenais F, Voisine P, Mathieu P. Giant pseudoaneurysm after proximal aortic surgery treated by means of redo axillary artery cannulation and use of an Endoclamp device. J Thorac Cardiovasc Surg 2005; 130: 208-209.
3. Rashed A, Gombocz K, Vigh A, Alotti N. Total proximal anastomosis detach ment after classical bentall procedure. Int I Surg Case Rep 2017; 37: 173-176.

4. Hasan SB, Khan FW, Hashmi S, Tariq M, Khan G. Repair of ascending aortic pseudoaneurysm eroding through the sternum. Asian Cardiovasc Thorac Ann 2019; 27: 36-38.

5. Estrera AL, Miller CC III, Porat E, Mohamed S, Kincade R, Huynh TT, Safi HJ. De terminants of early and late outcome for reoperations of the proximal aorta. Ann Thorac Surg 2004; 78: 837-845. 